Chapter 12

\title{
Kalman Filter for Moving Object Tracking: Performance Analysis and Filter Design
}

\author{
Kenshi Saho
}

Additional information is available at the end of the chapter

http://dx.doi.org/10.5772/intechopen.71731

\begin{abstract}
This chapter presents Kalman filters for tracking moving objects and their efficient design strategy based on steady-state performance analysis. First, a dynamic/measurement model is defined for the tracking systems, assuming both position-only and position-velocity measurements. Then, problems with the Kalman filter design in tracking systems are summarized, and an efficient steady-state performance index proposed by the author [termed the root-mean-squared error index (the RMS index)] is introduced to resolve these concerns. The analytical relationship between the proposed RMS index and the covariance matrix of the process noise is shown, leading to a proposed design strategy that is based on this relationship. Theoretical performance analysis is conducted using the performance indices to show the optimality of the design strategy. Numerical simulations show the validity of the theoretical analyses and effectiveness of the proposed strategy in realistic situations. In addition, the optimal performance of the position-only-measured and position-velocity-measured systems is analyzed and compared. This comparison shows that the position-velocity-measured Kalman filter tracking is accurate when compared with the position-only-measured filter.
\end{abstract}

Keywords: Kalman tracking filter, moving object tracking, steady-state analysis, performance index, filter design, process noise

\section{Introduction}

Remote monitoring systems for cars and robots require accurate tracking of moving objects. Representative tracking algorithms include the Kalman filter [1-5] and its variants, such as the extended/unscented Kalman [6-9] and particle filters [10-12]. These can accurately track movement based on adaptive filtering by using a state-space model.

To use the Kalman filter for the tracking of moving objects, it is necessary to design a dynamic model of target motion. The most common dynamic model is a constant velocity (CV) model 
$[1,10]$, which assumes that the velocity is constant during a sampling interval. This model has been used in many applications because of its versatility, effectiveness, and simplicity. However, in almost conventional tracking systems, the selection of process noise (zero-mean white noise in the dynamic model) is conducted empirically $[4,6,8]$. This is because conventional studies tend to assume that process noise takes one of a limited number of forms, which is known as appropriate selections. Thus, despite the large number of investigations into Kalman filter trackers, the optimal selection of a process noise model has not been discussed. The general problems of model selection for Kalman filter trackers were discussed by Ekstrand in 2012 [1]. In the years since, further research on these issues has been conducted, but no satisfactory solutions to the abovementioned problems have been presented. Crouse [13] described a general solution for optimal trackers in a steady state. However, this method also requires an empirical selection of the dynamic models. A detailed analysis of the Kalman filter has been provided for various applications, including global navigation satellite systems [14] and video trackers [15]. However, only limited systems have yet been considered, and no definitive parameter-setting procedure for the Kalman tracking filter has been provided. Although various criteria have been proposed and investigated for the design of Kalman filters and its variants to achieve better tracking accuracy, robustness, and real-time capability, relationship between these performance indices and the model parameters such as the process noise variance is not discussed even in recent studies [16].

Another significant problem in a measurement model of the conventional Kalman tracking filter is that most studies consider only position measurements and therefore cannot make full use of modern sensors that are able to measure velocity, such as ultrawideband Doppler radar $[17,18]$. Moreover, sensor fusion based on Internet of Things technology also enables the simultaneous measurement of position and velocity (e.g., sensor data fusion based on the communication between radars/lasers/sonars and speedometers embedded in targets). Consequently, Kalman filters for such systems have become an important area of research [19-24]. In Ref. [24], the extended Kalman filter for radar measurements is modified for range (position) and range-rate (velocity) measurements, and its effectiveness in realistic radar applications is verified. However, concrete design criterion is not shown. The number of conventional studies on position-velocity-measured (PVM) Kalman filters is smaller than those on the more common position-only-measured (POM) Kalman filters, and the performance and design of PVM Kalman filters are not sufficiently considered.

To resolve the two problems described above concerning the process noise selection and PVM systems, our previous work clarified the fundamental properties of PVM tracking filters [25, 26] and generated an efficient performance index to design an optimal process noise matrix [3, 5]. In the studies of PVM tracking filters [25, 26], fixed-gain PVM filter properties were analytically clarified, but there was no optimization of the PVM Kalman filters. In our work on the process noise matrix [3], an optimal POM Kalman filter, with respect to position prediction, was presented. In this chapter, an appropriate process noise design strategy, based on our proposed efficient steady-state performance index (introduced in Section 3), and its applicability are verified. Our previous work highlighted the following issues, which we address in this chapter: 
i. Analysis of the performance of a PVM Kalman filter with a CV model, based on the proposed index.

ii. Application of the proposed process noise design strategy to a PVM Kalman filter.

iii. Comparison of the performance of optimal POM and PVM Kalman filters.

This chapter presents the theoretical analyses and simulations required to tackle these issues. The remainder of this chapter is organized as follows: Section 2 defines the tracking filtering problem dealt in this chapter and explains the existing concerns and models for POM and PVM Kalman filter design. Section 3 introduces our proposed efficient performance indices with their mathematical formulations. Section 4 presents the proposed process noise design strategy based on the performance index. Section 5 shows the theoretical analysis of the optimal POM and PVM Kalman filter performance in a steady state. The effectiveness of the PVM Kalman filter is proven by the comparison with the POM filter. Section 6 shows realistic maneuvering-targettracking application examples. Section 7 concludes this chapter and proposes future tasks.

\section{Problem statement}

This section introduces the Kalman filter for moving object tracking and defines the model assumed in this chapter.

\subsection{Dynamic model}

The Kalman filter for tracking moving objects estimates a state vector comprising the parameters of the target, such as position and velocity, based on a dynamic/measurement model. For simplicity, this chapter deals with a typical second-order one-dimensional Kalman filter tracker whose true state vector is defined as

$$
\mathbf{x}_{\mathrm{t}}=\left(\begin{array}{ll}
x_{\mathrm{t}} & v_{\mathrm{t}}
\end{array}\right)^{\mathrm{T}},
$$

where $x_{\mathrm{t}}$ and $v_{\mathrm{t}}$ are the true position and velocity of the target moving object, respectively, and ${ }^{\mathrm{T}}$ denotes the transpose. The assumed dynamic model is a CV model, which is a simple and popular model for tracking moving objects. The CV model assumes that the velocity is constant during the sampling interval, which is expressed as

$$
\mathbf{x}_{\mathrm{t} k}=\boldsymbol{\Phi} \mathbf{x}_{\mathrm{t} k-1}+\mathbf{w}_{k},
$$

where $\mathbf{x}_{\mathrm{t} k}$ denotes the true state at time $k T, T$ is the sampling interval, $\mathbf{w}_{k}$ is the process noise with covariance matrix $\mathbf{Q}$, and $\boldsymbol{\Phi}$ is the transition matrix from $k T$ to $(k+1) T$, which is expressed as

$$
\boldsymbol{\Phi}=\left(\begin{array}{ll}
1 & T \\
0 & 1
\end{array}\right)
$$

The Kalman filter predicts the target state based on this dynamic model. 


\subsection{Measurement model}

The measurements are simply modeled as

$$
\mathbf{z}_{k}=\mathbf{H} \mathbf{x}_{\mathrm{t} k}+\mathbf{v}_{k}
$$

where $\mathbf{z}_{k}$ denotes the measurement vector, $\mathbf{H}$ denotes the measurement matrix, and $\mathbf{v}_{k}$ is the measurement noise with covariance matrix $\mathbf{R}$. This chapter considers two types of measurement systems, which are discussed as follows.

\subsubsection{Position-only-measured system}

The POM system assumes that the sensors (such as radar, laser, and sonar) can measure only the position of the target. This is a general assumption in the moving object tracking. $\mathbf{H}$ and $\mathbf{R}$ of this model are expressed as

$$
\begin{gathered}
\mathbf{H}=\left(\begin{array}{ll}
1 & 0
\end{array}\right), \\
\mathbf{R}=\left(B_{\mathbf{x}}\right),
\end{gathered}
$$

where $B_{\mathrm{x}}$ is the variance of the position measurement errors.

\subsubsection{Position-velocity-measured system}

The PVM system assumes that the sensor system can measure position and velocity simultaneously. One example of the PVM model system is a pulse Doppler radar. Sensor fusion systems using communications of position/velocity sensors can also be expressed by the PVM model. $\mathbf{H}$ of this model is expressed as

$$
\mathbf{H}=\left(\begin{array}{ll}
1 & 0 \\
0 & 1
\end{array}\right) .
$$

We now assume that the noises of position and velocity measurements are uncorrelated, and $\mathbf{R}$ of PVM systems under this assumption is defined as

$$
\mathbf{R}=\left(\begin{array}{ll}
B_{\mathrm{x}} & 0 \\
0 & B_{\mathrm{v}}
\end{array}\right)
$$

where $B_{\mathrm{v}}$ is the variance of the velocity measurement errors.

\subsection{Kalman filter tracking}

The Kalman filter tracker based on the abovementioned models sequentially estimates state vectors via the Kalman filter equations. The prediction and estimation are calculated as 


$$
\begin{gathered}
\tilde{\mathbf{x}}_{k}=\boldsymbol{\Phi} \widehat{\mathbf{x}}_{k-1}, \\
\widehat{\mathbf{x}}_{k}=\tilde{\mathbf{x}}_{k}+\mathbf{K}_{k}\left(\mathbf{z}_{k}-\mathbf{H} \tilde{\mathbf{x}}_{k}\right),
\end{gathered}
$$

where predicts and estimates are denoted by ${ }^{\sim}$ and ${ }^{\wedge}$, respectively, and $\mathbf{K}_{k}$ denotes the Kalman gain that minimizes the errors in the estimated position and velocity. $\mathbf{K}_{k}$ is calculated as

$$
\mathbf{K}_{k}=\tilde{\mathbf{P}}_{k} \mathbf{H}^{\mathrm{T}}\left(\mathbf{H} \tilde{\mathbf{P}}_{k} \mathbf{H}^{\mathrm{T}}+\mathbf{R}\right) .
$$

where $\mathbf{P}_{k}$ is the covariance matrix of errors determined from

$$
\begin{gathered}
\tilde{\mathbf{P}}_{k}=\boldsymbol{\Phi} \widehat{\mathbf{P}}_{k-1} \boldsymbol{\Phi}^{\mathrm{T}}+\mathbf{Q} . \\
\widehat{\mathbf{P}}_{k}=\tilde{\mathbf{P}}_{k}-\mathbf{K}_{k} \mathbf{H} \tilde{\mathbf{P}}_{k} .
\end{gathered}
$$

\subsection{Aspects of tracking filter design}

Moving object tracking obtains accurate and sequential estimation of the target position and velocity by using Eqs. (9)-(13). As indicated in Eqs. (1)-(13), the design parameters of the Kalman filter tracker are elements of the covariance matrix of the process noise $\mathbf{Q}$. We must set $\mathbf{Q}$ to achieve tracking errors that are as small as possible. Thus, we must know how to design an appropriate $\mathbf{Q}$. Moreover, we must be able to define the evaluation index of the filter performance. However, these issues have not been sufficiently deliberated because the selection of $\mathbf{Q}$ has not been sufficiently addressed in previous studies. Here, the design of $\mathbf{Q}$ is empirically carried out.

In the conventional tracking systems, the most commonly used random acceleration (RA) process noise is often selected because it has a better performance. Its $\mathbf{Q}$ is

$$
\mathbf{Q}_{\mathrm{ra}}=\left(\begin{array}{ll}
T^{4} / 4 & T^{3} / 2 \\
T^{3} / 2 & T^{2}
\end{array}\right) \sigma_{\mathrm{q}}^{2} .
$$

The appropriate selection of $\sigma_{\mathrm{q}}$ is important because $\sigma_{\mathrm{q}}$ (and sensor noise variance $\mathbf{R}$ ) directly determines the performance of the tracking filter with the CV model. However, in conventional studies, process noises and their parameters are empirically selected, and the validity of the selection is discussed only casually $[1,16]$. Many conventional tracking systems select the RA process noises $\left(\mathbf{Q}_{\mathrm{ra}}\right)$, with variance $\sigma_{\mathrm{q}}$ set based on the assumed target motion. However, no definitive method of determining $\sigma_{\mathrm{q}}$ has been established. Although tracking index defined by Kalata [27] is known as an effective design parameter, its empirical selection is still required. Moreover, the validity in selecting the RA process noise is also questionable. Various other forms of $\mathbf{Q}$ are known and have been used for different target motions [12]. For example, random velocity model [2] and the diagonal $\mathbf{Q}$, which do not include correlations in process noise [7], are also frequently used. However, for the reasons discussed earlier, the differences in performance between the various process noise models are not known. 


\section{The efficient steady-state performance index (RMS index)}

The process noise selection problems discussed in Section 2.3 must be solved to effectively design Kalman tracking filters. Thus, we must properly evaluate the performance of the filter. The effective steady-state performance index was derived [3] and is termed root-mean-squared error index (an RMS index). This section introduces the RMS index for POM and PVM systems and shows the analytical relationships between the RMS index and $\mathbf{Q}$.

\subsection{Definition of RMS index}

In tracking filtering, the following two functions are required:

- Function 1. Reduces random errors caused by measurement noises.

- Function 2. Tracks targets with complicated motions (e.g., accurate tracking of an accelerating target is required for the $\mathrm{CV}$ model).

The RMS index is proposed for the comprehensive evaluation of the performance of these two functions and is defined as

$$
\varepsilon_{\mathrm{p}} \equiv \sqrt{\mathrm{E}\left[\left(x_{\mathrm{tak}}-\tilde{x}_{k}\right)^{2}\right]}
$$

where $\tilde{x}_{k}$ is the predicted target position (second element of $\tilde{\mathbf{x}}_{k}$ ), E[] indicates the mean with respect to $k$, and $x_{\text {tak }}$ is the true position of a constant acceleration target which is

$$
x_{\mathrm{tak}}=a_{\mathrm{c}}(k T)^{2} / 2
$$

where $a_{\mathrm{c}}$ is constant acceleration of the target. In the Kalman filter tracker using the CV model, it is assumed that the target velocity is constant during the sampling interval. Thus, for the constant acceleration target, a steady-state bias error occurs because of the difference between the target motion and the assumed dynamic model. Moreover, $\tilde{x}_{k}$ includes random errors due to measurement noise. Thus, the RMS index $\varepsilon_{\mathrm{p}}$ expresses both bias errors and random errors. With the steady-state bias error due to the model/motion difference of $e_{\mathrm{ac}}$ and the steady-state standard deviation of the random errors in $\tilde{x}_{k}$ of $\sigma_{\mathrm{p}}, \varepsilon_{\mathrm{p}}$ is expressed as

$$
\varepsilon_{\mathrm{p}}=\sqrt{e_{\mathrm{ac}}^{2}+\sigma_{\mathrm{p}}^{2}}
$$

$\sigma_{\mathrm{p}}$ expresses the performance corresponding to Function 1 and $e_{\mathrm{ac}}$ expresses the performance corresponding to Function 2. The smaller these errors are, the better is the tracking filter. Thus, the minimum $\varepsilon_{\mathrm{p}}$ achieves the best tracking filter in a steady state.

\subsection{RMS index of a POM system}

One important advantage of the RMS index is that it can be expressed in closed form. The closed form of $\varepsilon_{\mathrm{p}}$ for the POM system was derived in Ref. [3]. This subsection introduces the RMS index and its relationship to the design parameter $\mathbf{Q}$ in the POM system. 
First, the arbitrary $\mathbf{Q}$ is defined as

$$
\mathbf{Q}_{\text {gen }}=\left(\begin{array}{ll}
a & b \\
b & c
\end{array}\right)
$$

where $a>0, b>0$, and $c>0$, and the dimensions of $a, b$, and $c$ are $\left[\mathrm{m}^{2}\right],\left[\mathrm{m}^{2} / \mathrm{s}\right]$, and $\left[\mathrm{m}^{2} / \mathrm{s}^{2}\right]$, respectively. For example, substituting $(a, b, c)=\left(\sigma_{\mathrm{q}}^{2} T^{4} / 4, \sigma_{\mathrm{q}}^{2} T^{3} / 2, \sigma_{\mathrm{q}}^{2} T^{2}\right)$ into Eq. (18) gives the $\mathbf{Q}_{\mathrm{ra}}$ of Eq. (14) and $b=0$ leads to the diagonal $\mathbf{Q}$. The analytical relationship between $\mathbf{Q}_{\mathrm{gen}}$ and $\varepsilon_{\mathrm{p}}$ is expressed by the following closed form.

$$
\varepsilon_{\mathrm{p}, \mathrm{pom}}^{2}=\frac{a_{\mathrm{c}}^{2} T^{4}}{\beta^{2}}+\frac{2 \alpha^{2}+2 \beta+\alpha \beta}{\alpha(4-2 \alpha-\beta)} B_{\mathrm{x}}
$$

where $\alpha$ and $\beta$ are components of the steady-state Kalman gain $\mathbf{K}_{\infty}=(\alpha, \beta / T)^{\mathrm{T}}$ calculated from $(a, b, c)$ using the following equations:

$$
\begin{gathered}
\beta=\frac{C+\sqrt{C(16+4 A-4 B+C)}}{4}-\sqrt{\frac{C^{2}(16+4 A-4 B+C)}{8 \sqrt{C(16+4 A-4 B+C)}}+\frac{C(2 A-2 B+C}{8}} \\
A=a / B_{\mathrm{x}}, \quad B=b T / B_{\mathrm{x}}, \quad C=c T^{2} / B_{\mathrm{x}} \\
\alpha=1-\beta^{2} / C
\end{gathered}
$$

The derivation process of these equations is shown in Ref. [3]. As shown in Eqs. (19)-(22), the optimal $(a, b, c)$ is designed minimizing $\varepsilon_{\mathrm{p}}$.

\subsection{RMS index of a PVM system}

In a similar manner to the treatment of the POM system, this subsection introduces the RMS index of a PVM system and its relationship to $\mathbf{Q}_{\text {gen }}$. The RMS index of the PVM system is

$$
\varepsilon_{\mathrm{p}, \mathrm{pvm}}^{2}=\left(\frac{2-2 \eta-\theta}{2(\beta+\alpha \theta-\beta \eta)}\right)^{2} a_{\mathrm{c}}^{2} T^{4}+\frac{g_{2}(\alpha, \beta, \eta, \theta) B_{\mathrm{x}}+g_{3}(\alpha, \beta, \eta, \theta) T^{2} B_{\mathrm{v}}}{g_{1}(\alpha, \beta, \eta, \theta)}
$$

where $\alpha, \beta, \eta$, and $\theta$ are components of the steady-state Kalman gain:

$$
\mathbf{K}_{\infty}=\left(\begin{array}{ll}
\alpha & T \eta \\
\beta / T & \theta
\end{array}\right)
$$

and,

$$
g_{1}(\alpha, \beta, \eta, \theta)=(\beta \eta-\alpha \theta-\beta)(\alpha \theta-\beta \eta-\alpha-\theta)(4-2 \alpha-\beta-2 \theta+\alpha \theta-\beta \eta)
$$




$$
\begin{aligned}
g_{2}(\alpha, \beta, \eta, \theta) & =\alpha^{3} \theta(\theta-2)(\theta-1)+\alpha^{2} \beta\left(2-2 \theta-3 \eta \theta^{2}+6 \eta \theta-2 \eta\right) \\
& +\alpha^{2} \theta^{2}(2-\theta)+2 \alpha \beta \theta(\eta-2)(\theta-2)+\alpha \beta^{2}\left(1+2 \eta-\theta-3 \eta^{2}+3 \eta^{2} \theta\right) \\
& +\beta^{3} \eta(1+\eta)(1-\eta)+\beta^{2} \eta(2-\eta)(\theta-2)+\beta^{2}(2-\theta) \\
g_{3}(\alpha, \beta, \eta, \theta)= & \alpha \theta\left(2 \eta^{2}+2 \eta \theta+\theta^{2}-\theta\right)+\eta \beta\left(2 \eta+2 \theta-2 \eta^{2}-\theta^{2}-2 \eta \theta\right)+\theta^{2}(2-\theta)
\end{aligned}
$$

Eq. (22) is obtained from $\sigma_{\mathrm{p}}^{2}$ and $e_{\mathrm{ac}}$ of the steady-state PVM Kalman filter $(\alpha-\beta-\eta-\theta$ filter) by using Eq. (17). The derivation processes for these are shown in Ref. [25]. The relationship between the steady-state Kalman gains and $\mathbf{Q}_{\text {gen }}$ is derived as follows:

$$
\begin{gathered}
a=\frac{T^{2} B_{\mathrm{v}}}{1-R_{\mathrm{xv}} \beta^{2}-(1-\theta) \alpha-\theta}\left\{\left(\alpha \beta^{2}+2 \beta^{3}+\beta^{2}+(\theta-1) \theta \beta\right) R_{\mathrm{xv}}\right. \\
\left.+\alpha^{2} R_{\mathrm{xv}}\left((1-\theta)(1+2 \alpha \beta)+\beta^{2} \theta+\beta\left(3 \theta-2-\theta^{2}\right)\right)+\theta(1-\theta)(\alpha-1)\right\} \\
b=\frac{\beta^{3} R_{\mathrm{xv}}^{2}+R_{\mathrm{xv}}\left(\alpha\left(\beta(1-\theta)-\theta^{2}+\theta\right)+\beta^{2} \theta+\beta \theta+\theta^{2}-\theta\right)}{1-R_{\mathrm{xv}} \beta^{2}-(1-\theta) \alpha-\theta} T B_{\mathrm{v}} \\
c=\frac{R_{\mathrm{xv}}\left(\alpha \beta \theta+\beta^{2}(\theta+1)-\beta \theta\right)-\alpha \theta(\beta+\theta)+\beta \theta+\theta^{2}}{1-R_{\mathrm{xv}} \beta^{2}-(1-\theta) \alpha-\theta} B_{\mathrm{v}}
\end{gathered}
$$

where

$$
\begin{gathered}
R_{\mathrm{xv}}=B_{\mathrm{x}} / T^{2} B_{\mathrm{v}} \\
\eta=R_{\mathrm{xv}} \beta
\end{gathered}
$$

The derivation of these is given in the Appendix. Note that the dimensionless parameter $R_{\mathrm{xV}}$ corresponds to the ratio of the measurement accuracies in position and velocity and directly affects the tracking accuracy in PVM tracking systems. From these results, we also obtain the closed form of the RMS index for PVM systems and can design optimal $\mathbf{Q}$ using Eqs. (22)-(32).

\section{Filter design strategy based on the RMS index}

Using the RMS index introduced in the previous section, we can design the Kalman filter parameters (i.e., $\mathbf{Q}$ ) to achieve optimal tracking. This section defines the optimization problems for POM and PVM systems with a $\mathbf{Q}$ that minimizes the RMS index $\varepsilon_{\mathrm{p}}$.

\subsection{RMS-index minimization problem}

\subsubsection{POM system optimization}

The evaluating function to determine optimal $\mathbf{Q}$ is $\varepsilon_{\mathrm{p}, \text { pom }}$ normalized by $B_{\mathrm{x}}$ which is defined as 


$$
\mu_{\mathrm{pom}}=\varepsilon_{\mathrm{p}, \mathrm{pom}}^{2} / B_{\mathrm{x}}=\frac{a_{\mathrm{D}}^{2}}{\beta^{2}}+\frac{2 \alpha^{2}+2 \beta+\alpha \beta}{\alpha(4-2 \alpha-\beta)}
$$

where

$$
a_{\mathrm{D}}^{2}=a_{\mathrm{c}}^{2} T^{4} / B_{\mathrm{x}}
$$

is the preset parameter for the proposed strategy. Substituting Eqs. (20)-(22) into (33), we obtain $\mu_{\text {pom }}\left(a, b, c, a_{\mathrm{D}}\right)$. Using this, the optimal $(a, b, c)$ for the POM system is determined by solving

$$
\begin{array}{ll}
\arg \min _{a, b, c} & \mu_{\text {pom }}\left(a, b, c, a_{\mathrm{D}}\right) \\
\text { sub. to. } & a>0, b>0, c>0, \text { and } a_{\mathrm{D}}=\text { Const. }
\end{array}
$$

\subsubsection{PVM system optimization}

Like the POM system, a normalized RMS index can be used for the design of the PVM system. Normalizing Eq. (22) by $B_{x}$ and substituting Eqs. (31) and (32) into this, the evaluating function for the PVM system is given by

$$
\mu_{\mathrm{pvm}}=\varepsilon_{\mathrm{p}, \mathrm{pvm}}^{2} / B_{\mathrm{x}}=\left(\frac{2-2 \beta R_{\mathrm{xv}}-\theta}{2\left(\beta+\alpha \theta-\beta^{2} R_{\mathrm{xv}}\right)}\right)^{2} a_{\mathrm{D}}^{2}+\frac{g_{2}\left(\alpha, \beta, \theta, R_{\mathrm{xv}}\right)+g_{3}\left(\alpha, \beta, \theta, R_{\mathrm{xv}}\right) / R_{\mathrm{xv}}}{g_{1}\left(\alpha, \beta, \theta, R_{\mathrm{xv}}\right)}
$$

To design optimal $(a, b, c)$ for the PVM system, the optimal steady-state Kalman gains are calculated by solving the following minimization problem.

$$
\begin{array}{ll}
\arg \min _{\alpha, \beta, \theta} & \mu_{\mathrm{pvm}}\left(\alpha, \beta, \theta, R_{\mathrm{xv}}\right) \\
\text { sub. to. } & \text { Stability conditions are satisfied, and } a_{\mathrm{D}}=\text { Const. }
\end{array}
$$

where the stability conditions with respect to Kalman gains are easily derived by the wellknown Jury's test as

$$
(1-\eta) \beta<\alpha \theta \text { and } 4-2 \alpha-\beta-2 \theta+\alpha \theta-\eta \beta>0 \text { and }|\alpha \theta-\eta \beta-\alpha-\theta+1|<1
$$

Substituting the optimal $(\alpha, \beta, \theta)$ calculated by Eq. (37) into Eqs. (28)-(30), we obtain an optimal $(a, b, c)$ for the PVM Kalman filter.

\subsection{Procedure and notes of the proposed design strategy}

The procedure of the proposed strategy for each system is summarized in this section.

\subsubsection{Design procedure for a POM system}

1. Set $B_{\mathrm{x}}$ from the sensor performance. 
2. Preset $a_{\mathrm{D}}$ based on the approximate target acceleration.

3. Determine $(a, b, c)$ by solving Eq. (35).

The methodology of presetting $a_{\mathrm{D}}$ is discussed in the simulation section.

\subsubsection{Design procedure for a PVM system}

1. Set $B_{\mathrm{x}}$ and $B_{\mathrm{v}}$ from the sensor performance.

2. Preset $a_{\mathrm{D}}$ based on the approximate target acceleration.

3. Determine $(\alpha, \beta, \theta)$ by solving Eq. (37).

4. Determine $(a, b, c)$ from $(\alpha, \beta, \theta)$ using Eqs. (28)-(30).

\subsubsection{Notes on computation in the proposed strategy}

With respect to the proposed strategy, note that:

- $\quad$ Eqs. (35) and (37) can be solved by gradient descent with several initial values. This is because that the parameter searching range is narrow due to the stability conditions.

- The proposed design process is only carried out once before using the Kalman filter. Although the computational cost of the above optimization process is not small, it does not affect the Kalman filtering process.

\subsection{Discussion on preset parameter $a_{\mathrm{D}}$}

Here, the appropriate presetting for $a_{\mathrm{D}}$ in practical use is discussed. The covariance matrix of process noise $\mathbf{Q}$ determined by the proposed strategy is only optimal when $a_{\mathrm{D}}$ is matched to the target acceleration and the target is moving with constant acceleration corresponding to $a_{\mathrm{D}}$. However, using the proposed strategy, the tracking accuracy is always better than when using conventional models as verified in Ref. [3]. Consequently, the proposed method achieves sufficient accuracy, even if $a_{\mathrm{D}}$ is not matched to the true target acceleration. This means that the relatively small difference between the true and preset acceleration is acceptable. Thus, in practical use, we estimate an approximate or a typical value for the acceleration (e.g., mean and maximum) in advance based on the assumed motion of the target and then set $a_{\mathrm{D}}$ by using this estimated value. The example application presented in Section 6 assumes the approximate maximum acceleration of the target is known and is used for the Kalman filter design.

Thus, target acceleration information is required for accurate Kalman filter tracking by using the proposed strategy. As a method to obtain an approximated acceleration, communications between the tracking systems and the accelerometers embedded in targets can be considered. Many sensing targets have acceleration sensors; for example, robots and vehicles have inertial sensors, and humans have accelerometers embedded in smartphones. Soon, Internet of Things 
technology will make data communications between robots, smartphones, and radar possible. Thus, we can obtain approximated acceleration based on this novel technology.

\section{Theoretical steady-state performance analysis}

This section presents theoretical performance analyses of the Kalman tracking filters by using the proposed design strategy. With respect to POM systems, our previous study [3] verified the effectiveness of the proposed strategy by comparison with a conventional random acceleration model based filter design. Thus, the RMS indices for the following filters are compared:

- Optimal POM filter: the Kalman filter for the POM system designed using the strategy mentioned in Section 4.2.1.

- Optimal PVM filter: the Kalman filter for the PVM system designed using the strategy mentioned in Section 4.2.2.

- $\quad$ RA filter: the Kalman filter for the PVM system with the RA process noises by using optimal $\sigma_{\mathrm{q}}$ with respect to the RMS index.

The comparison of the optimal PVM filter with the RA filter indicates the effectiveness of the proposed strategy (i.e., considering the arbitrary covariance matrix of the process noise $\mathbf{Q}_{\text {gen }}$ ) and the comparison of the optimal POM and PVM filters illustrates the enhancement of tracking accuracy by using the velocity measurements in the proposed strategy. This section assumes that $B_{\mathrm{x}}$ and $T$ are normalized to 1 .

Figure 1 shows the relationship between the design parameter $a_{\mathrm{D}}$ and the minimum RMS index $\varepsilon_{\mathrm{p} \text {, opt }}$ for $R_{\mathrm{xv}}=1$ (Figure 1 left) and $R_{\mathrm{xV}}=10$ (Figure 1 right). It can be seen that the optimal PVM filter achieves the best performance. This result verifies that the proposed strategy determines steady-state gains corresponding to a better covariance matrix of process noise than the RA model. The optimal PVM filter also achieves better performance compared with the optimal POM filter even for $R_{\mathrm{xv}}=1$, which means that the measurement accuracy of the
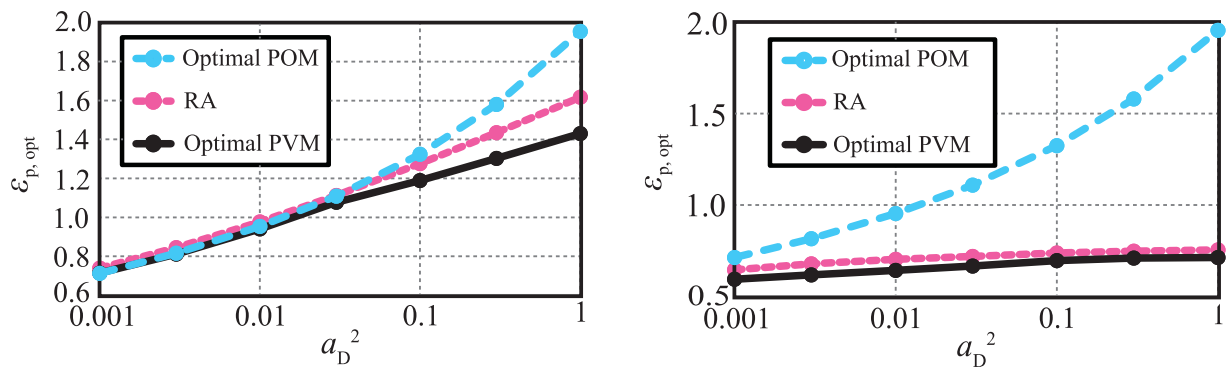

Figure 1. Analytical relationship between $a_{\mathrm{D}}$ and $\varepsilon_{\mathrm{p}, \mathrm{opt}}\left(R_{\mathrm{xv}}=1\right.$ (left), $R_{\mathrm{xv}}=10$ (right)). 

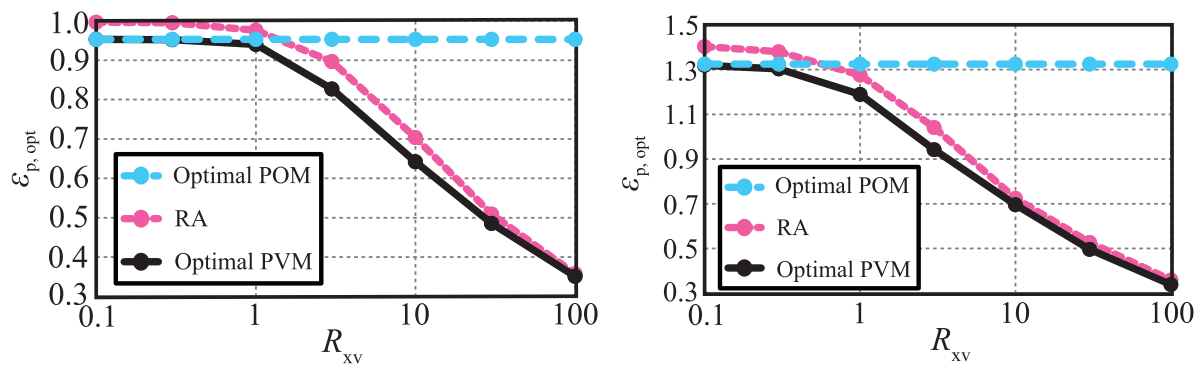

Figure 2. Analytical relationship between $R_{\mathrm{xv}}$ and $\varepsilon_{\mathrm{p} \text {, opt }}\left(a_{\mathrm{D}}=0.01\right.$ (left), $a_{\mathrm{D}}=0.1$ (right)).

position and velocity is the same. The addition of the velocity measurements effectively enhances the tracking accuracy. Furthermore, when the velocity measurement accuracy is high, the optimal PVM filter achieves greater accuracy than the POM filter.

Figure 2 shows the relationship between $R_{\mathrm{xv}}$ and $\varepsilon_{\mathrm{p} \text {, opt }}$ for $a_{\mathrm{D}}^{2}=0.01$ (left) and 0.1 (right). Both cases exhibit the same trend. For both optimal PVM and RA filters, better performance is achieved with better velocity measurement accuracy. The performance of the optimal PVM filter is better than that of the optimal POM filter including relatively small $R_{\mathrm{xV}}$ (the velocity measurement accuracy is low). In contrast, the performance of the RA filter is worse than that of the optimal POM filter for small $R_{\mathrm{xv}}$ because the covariance matrix is limited to Eq. (14). Moreover, by comparing the two insets of Figure 2, we see the greater effectiveness of the proposed strategy for relatively large $a_{\mathrm{D}}$.

\section{Application to radar tracking simulation}

Finally, this section provides an example of the Kalman filter tracker designed with the proposed strategy in a realistic application, namely, pulse Doppler radar tracking.

\subsection{Simulation setup}

We simulated the pulse Doppler radar tracking of a maneuvering target and compared the tracking errors of the filters assumed in the previous section. Figure 3 shows the simulation scenario and the true target acceleration. The true target position is $\left(x_{\mathrm{t} k}, y_{\mathrm{t} k}\right)=\left((k T)^{2}, 20+\right.$ $\left.(k T)^{1.5} \cos (\pi k T / 5)\right)$. Two-dimensional tracking in the $x-y$ plane of the point target is assumed. We consider two pulse Doppler radars located at $(x, y)=(0.5 \mathrm{~m}, 0)$ and $(1.0 \mathrm{~m}, 0)$. The sampling interval $T$ is $100 \mathrm{~ms}$, and the observation time is $4 \mathrm{~s}$. The transmitted signal is a pulse with central frequency of $60 \mathrm{GHz}$ and bandwidth of $500 \mathrm{MHz}$. The received radar signals are calculated using ray tracing with the addition of the Gaussian white noise. The radar measurement parameter depends on the system under consideration: the POM system assumes the measurement of the position by using ranging results, and the PVM system assumes the position and velocity measurements where the position measurement is the same as the POM 

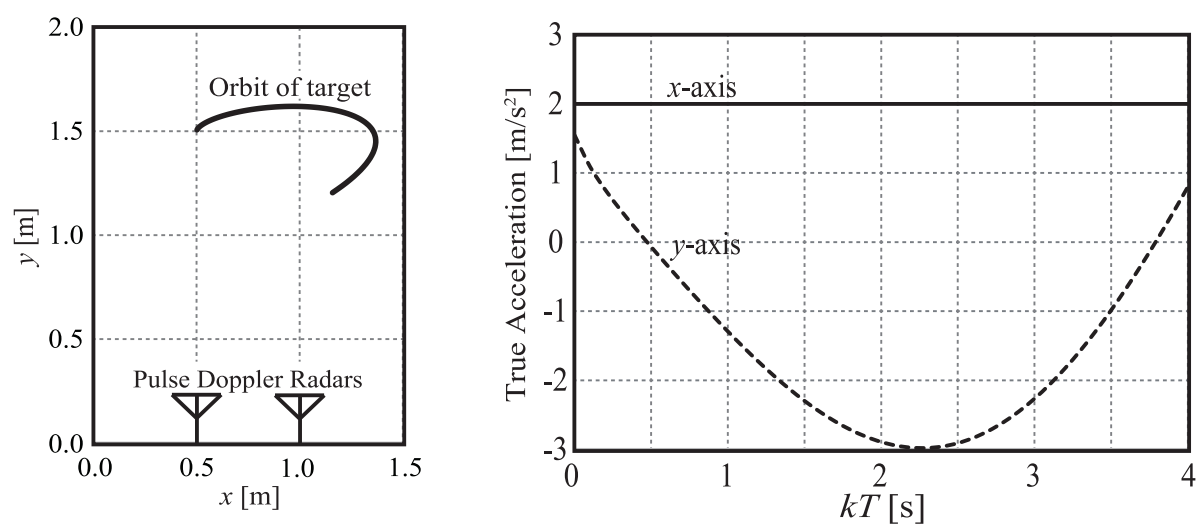

Figure 3. Simulation setting (simulation scenario (left), true target acceleration (right)).

system, and the velocity measurement is based on the Doppler shift with the method presented in Ref. [18]. We determine a variance for this noise to set $B_{\mathrm{x}}=9 \times 10^{-4} \mathrm{~m}^{2}$ and $B_{\mathrm{v}}=0.09 \mathrm{~m}^{2} / \mathrm{s}^{2}$. In these settings, $R_{\mathrm{xv}}=1$. These values are the averages along the two axes. Using the RMS prediction error calculated from 1000 Monte Carlo simulations, the performance is defined as

$$
\varepsilon_{k}=\sqrt{\frac{1}{1000} \sum_{m=1}^{1000}\left\{\left(x_{\mathrm{t} k}-x_{\mathrm{p} m k}\right)^{2}+\left(y_{\mathrm{t} k}-y_{\mathrm{p} m k}\right)^{2}\right\}}
$$

where $x_{\mathrm{p} m k}$ and $y_{\mathrm{p} m k}$ are the predicted positions in the $m$ th Monte Carlo simulation.

\subsection{Implementation of Kalman filter}

First, the implementation of the Kalman filters for two-dimensional system is presented. The implementation of a two-dimensional optimal POM filter is as follows:

$$
\begin{gathered}
\mathbf{x}_{\mathrm{t}}=\left(\begin{array}{llll}
x_{\mathrm{t}} & v_{\mathrm{xt}} & y_{\mathrm{t}} & v_{\mathrm{yt}}
\end{array}\right)^{\mathrm{T}} \\
\mathbf{\Phi}=\left(\begin{array}{llll}
1 & T & 0 & 0 \\
0 & 1 & 0 & 0 \\
0 & 0 & 1 & T \\
0 & 0 & 0 & 1
\end{array}\right) \\
\mathbf{H}=\left(\begin{array}{llll}
1 & 0 & 0 & 0 \\
0 & 0 & 1 & 0
\end{array}\right) \\
\mathbf{R}=\left(\begin{array}{lll}
B_{\mathrm{x}} & 0 \\
0 & B_{\mathrm{x}}
\end{array}\right)
\end{gathered}
$$




$$
\mathbf{Q}=\left(\begin{array}{llll}
a_{\mathrm{opt}} & b_{\mathrm{opt}} & 0 & 0 \\
b_{\mathrm{opt}} & c_{\mathrm{opt}} & 0 & 0 \\
0 & 0 & a_{\mathrm{opt}} & b_{\mathrm{opt}} \\
0 & 0 & b_{\mathrm{opt}} & c_{\mathrm{opt}}
\end{array}\right)
$$

where $v_{\mathrm{yt}}$ is the true velocity in the $y$-axis and $\left(a_{\mathrm{opt}}, b_{\mathrm{opt}}, c_{\mathrm{opt}}\right)$ is optimized $(a, b, c)$, calculated using the procedure in Section 4.2.1. $\mathbf{x}_{\mathrm{t}}$ and $\Phi$ of a two-dimensional PVM filter are the same as for a POM filter. $\mathbf{H}$ and $\mathbf{R}$ are

$$
\begin{aligned}
\mathbf{H} & =\left(\begin{array}{llll}
1 & 0 & 0 & 0 \\
0 & 1 & 0 & 0 \\
0 & 0 & 1 & 0 \\
0 & 0 & 0 & 1
\end{array}\right) \\
\mathbf{R} & =\left(\begin{array}{llll}
B_{\mathrm{x}} & 0 & 0 & 0 \\
0 & B_{\mathrm{v}} & 0 & 0 \\
0 & 0 & B_{\mathrm{x}} & 0 \\
0 & 0 & 0 & B_{\mathrm{v}}
\end{array}\right)
\end{aligned}
$$

In addition, the formulation of $\mathbf{Q}$ is the same as in Eq. (43) and $\left(a_{\mathrm{opt}}, b_{\mathrm{opt}}, c_{\mathrm{opt}}\right)$ is calculated using the procedure in Section 4.2.2. A two-dimensional RA filter is the same as the optimal PVM filter, with the exception of $\mathbf{Q}$. $\mathbf{Q}$ of the RA filter is

$$
\mathbf{Q}_{\text {ra }}=\left(\begin{array}{llll}
T^{4} / 4 & T^{3} / 2 & 0 & 0 \\
T^{3} / 2 & T^{2} & 0 & 0 \\
0 & 0 & T^{4} / 4 & T^{3} / 2 \\
0 & 0 & T^{3} / 2 & T^{2}
\end{array}\right) \sigma_{\mathrm{q}}^{2}
$$

Next, the design for an appropriate $a_{\mathrm{D}}$ is presented. We presume an approximate prediction of accelerations. For instance, when the maximum acceleration of the target in Figure 3 is predicted to be approximately $a_{\mathrm{c}}=3 \mathrm{~m} / \mathrm{s}^{2}, a_{\mathrm{D}}$ is then 1.0, from Eq. (34). Using this $a_{\mathrm{D}}$ and the radar settings described in the previous section, we have $\left(a_{\mathrm{opt}}, b_{\mathrm{opt}}, c_{\mathrm{opt}}\right)$ for each filter.

\subsection{Results and discussion}

Figure 4 shows the simulation results. Clearly, the filters using velocity measurements achieve greater accuracy than the optimal POM filter. The mean steady-state prediction RMS errors $\left(\mathrm{E}\left[\varepsilon_{k}\right]\right.$ in $2 \mathrm{~s}<k T$ ) of the optimal POM, RA, and optimal PVM filters are 0.59, 0.46, and $0.19 \mathrm{~m}$, respectively. These results indicate that the proposed strategy achieves greater accuracy than the conventional RA filter even in realistic situations. The mean RMS error of the optimal PVM filter is $41 \%$ of that of the RA filter. This is because the RA model cannot track the abrupt motion of the high-maneuvering target because of limitations in expressing the process noise. In contrast, the optimal PVM filter can set gains corresponding to the appropriate process 


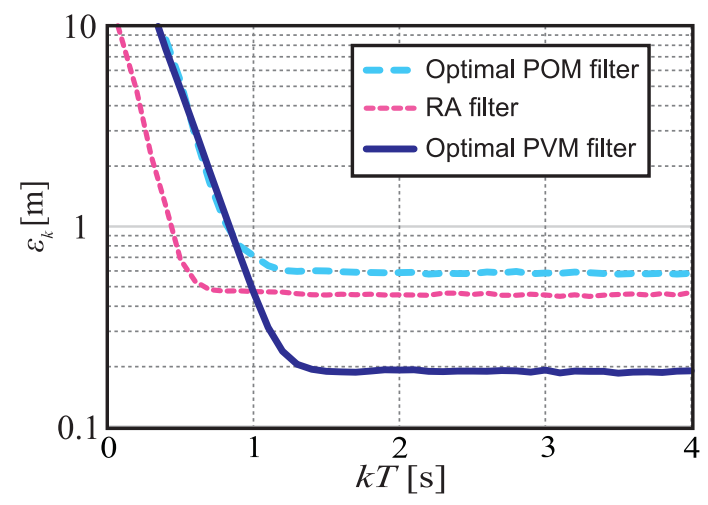

Figure 4. Simulation results.

noise to accurately track high-maneuvering target. Moreover, the mean RMS error of the optimal PVM filter is $32 \%$ of the error in optimal POM filter, and this clearly indicates the effectiveness of the velocity measurement, even when $R_{\mathrm{xv}}=1$ (when the measurement reliability of the position and velocity are the same). These simulation results are consistent with the theoretical analyses presented in Figure 1.

\section{Final remarks}

\subsection{Conclusions}

In this chapter, the efficient steady-state performance index, known as the RMS index, was introduced for both POM and PVM Kalman filters for systems that track moving objects. Automatic design (preset) of the covariance matrix of the process noise $\mathbf{Q}$, to realize optimal position prediction, was achieved using the analytical relationship between $\mathbf{Q}$ and the RMS index. The validity of the proposed design strategy was shown via analyses and simulations. These results verified that the proposed index attained accurate tracking when compared with the conventional RA-model-based Kalman filter design. A simulation of a realistic situation indicated that the optimal performance given by the proposed strategy is $41 \%$ better than that given by the conventional design procedure for a PVM system. Moreover, the optimal performance of the optimal POM and PVM Kalman filters was compared showing that the optimal PVM Kalman filter is accurate when compared with the POM filter in a steady state.

\subsection{Future works}

The most important future objective is the extension of the RMS index-based design strategy to the third-order (and higher order) Kalman filters that are widely used for real applications. In third-order tracking, an acceleration is added to the state vector, becoming one of the input parameters of the Kalman filter. Performance analysis and the establishment of a design 
strategy for such systems (i.e., position/acceleration and position/velocity/acceleration measured Kalman filters) are important considerations for advanced sensor fusion systems under development. Moreover, considerations of other dynamic models (e.g., the constant turn model) should also be probed for use in many applications including pedestrian tracking.

\section{A. Appendix}

\section{A.1. Derivation of Eqs. (28)-(30)}

Because we assume a steady state, the index $k$ of all parameters and matrices is omitted in the following calculations. The $i$ th row and $j$ th column of a matrix $\mathbf{P}$ are denoted as $P^{i, j}$.

Eq. (11) is also written using $\widehat{\mathbf{P}}$ as

$$
\mathbf{K}=\widehat{\mathbf{P}} \mathbf{H}^{\mathrm{T}} \mathbf{R}^{-1}
$$

As indicated in Eq. (7), $\mathbf{H}$ of the PVM Kalman filter is the identity matrix. Thus, from Eq. (48), the relationship between the Kalman gains and the error covariance matrix in the estimated state $\widehat{\mathbf{P}}$ is calculated using Eqs. (8) and (24) as

$$
\widehat{\mathbf{P}}=\mathbf{K} \mathbf{R}=\left(\begin{array}{ll}
\alpha B_{\mathrm{x}} & T \eta B_{\mathrm{v}} \\
\beta B_{\mathrm{x}} / T & \theta B_{\mathrm{v}}
\end{array}\right)
$$

With $P^{1,2}=P^{2,1}$ and Eq. (31), we have the following relationship:

$$
\eta=\beta \frac{B_{\mathrm{x}}}{T^{2} B_{\mathrm{v}}}=\beta R_{\mathrm{xv}}
$$

Eq. (50) is equal to Eq. (32), showing that this relationship is satisfied in the assumed PVM Kalman filter without depending on the process noise. $\widehat{\mathbf{P}}$ is also calculated using Eq. (13) by substituting Eqs. (7) and (24) as

$$
\widehat{\mathbf{P}}=\left(\begin{array}{ll}
(1-\alpha) \tilde{P}^{1,1}-\operatorname{T\eta } \tilde{P}^{1,2} & (1-\alpha) \tilde{P}^{1,2}-\operatorname{T\eta } \tilde{P}^{2,2} \\
(1-\theta) \tilde{P}^{1,2}-(\beta / \mathrm{T}) \tilde{P}^{1,1} & (1-\theta) \tilde{P}^{2,2}-(\beta / \mathrm{T}) \tilde{P}^{1,2}
\end{array}\right)
$$

Elements of $\tilde{\mathbf{P}}$ are required to calculate Eq. (51) and are calculated using Eqs. (3), (12), and (18) as

$$
\tilde{\mathbf{P}}=\left(\begin{array}{ll}
P^{1,1}+2 T P^{1,2}+T^{2} P^{2,2}+a & P^{1,2}+T P^{2,2}+b \\
P^{1,2}+T P^{2,2}+b & P^{2,2}+c
\end{array}\right)
$$

Substituting Eq. (52) into Eq. (51), and comparing elements of Eq. (49), we have the following linear system: 


$$
\begin{gathered}
\alpha B_{\mathrm{x}}=(1-\alpha)\left(\alpha B_{\mathrm{x}}+2 \eta T^{2} B_{\mathrm{v}}+\theta T^{2} B_{\mathrm{v}}+a\right)-\eta T\left(\eta T B_{\mathrm{v}}+\theta T B_{\mathrm{v}}+b\right) \\
\beta B_{\mathrm{x}} / T=(1-\alpha)\left(\eta T B_{\mathrm{v}}+\theta T B_{\mathrm{v}}+b\right)-\eta T\left(\theta B_{\mathrm{v}}+c\right) \\
\theta B_{\mathrm{v}}=(1-\theta)\left(\theta B_{\mathrm{v}}+c\right)-(\beta / T)\left(\eta T B_{\mathrm{v}}+\theta T B_{\mathrm{v}}+b\right)
\end{gathered}
$$

Solving this linear system with respect to $(a, b, c)$ and substituting Eq. (50) into the solutions, we arrive at Eqs. (28)-(30).

\section{Author details}

Kenshi Saho

Address all correspondence to: saho@pu-toyama.ac.jp

Department of Intelligent Systems Design Engineering, Toyama Prefectural University, Imizu, Toyama, Japan

\section{References}

[1] Ekstrand B. Some aspects on filter design for target tracking. Journal of Control Science and Engineering. 2012;2012:870890. DOI: 10.1155/2012/870890

[2] Bar-Shalom Y, Li XR, Kirubarajan T. Estimation and Tracking: Principles, Techniques, and Software. Boston, MA, United States: Artech House Publishers; 1998

[3] Saho K, Masugi M. Automatic parameter setting method for an accurate Kalman filter tracker using an analytical steady-state performance index. IEEE Access. 2015;3:1919-1930. DOI: 10.1109/ACCESS.2015.2486766

[4] Hashlamon I, Erbatur K. An improved real-time adaptive Kalman filter with recursive noise covariance updating rules. Turkish Journal of Electrical Engineering \& Computer Sciences. 2016;24:524-540. DOI: 10.3906/elk-1309-60

[5] Basso G, Amorim TG, Brito AV, Nascimento TP. Kalman filter with dynamical setting of optimal process noise. IEEE Access. 2017;5:8385-8393. DOI: 10.1109/ACCESS.2017.2697072

[6] Fan Y, Lu F, Zhu W, Bai G, Yan L. A hybrid model algorithm for hypersonic glide vehicle Maneuver tracking based on the aerodynamic model. Applied Sciences. 2017;7:159. DOI: 10.3390/app7020159

[7] Li W, Sun S, Jia Y, Du J. Robust unscented Kalman filter with adaptation of process and measurement noise covariances. Digital Signal Processing. 2016;48:93-103. DOI: 10.1016/j. dsp.2015.09.004 
[8] Vivone F, Braca P, Horstmann J. Knowledge-based multitarget ship tracking for HF surface wave radar systems. IEEE Transactions on Geoscience and Remote Sensing. 2015;53:39313949. DOI: $10.1109 /$ TGRS.2014.2388355

[9] Du G, Zhang P. A Markerless human robot Interface using particle filter and Kalman filter for dual robots. IEEE Transactions on Industrial Electronics. 2015;62:2257-2264. DOI: 10.1109/TIE.2014.2362095

[10] Martino L, Read J, Elvira V, Louzada F. Cooperative parallel particle filters for on-line model selection and applications to urban mobility. Digital Signal Processing. 2017;60: 172-185. DOI: 10.1016/j.dsp.2016.09.011

[11] Drovandi CC, McGree J, Pettitt AN. A sequential Monte Carlo algorithm to incorporate model uncertainty in Bayesian sequential design. Journal of Computational and Graphical Statistics. 2014;23:324. DOI: 10.1080/10618600.2012.730083

[12] Li XR, Jilkov VP. Survey of maneuvering target tracking. Part I: Dynamic models. IEEE Transactions on Aerospace and Electronic Systems. 2003;39:1333-1364 DOI: 10.1109/ TAES.2003.1261132

[13] Crouse DF. A general solution to optimal fixed-gain (alpha-beta-gamma etc.) filters. IEEE Signal Processing Letters. 2015;22:901-904. DOI: 10.1109/LSP.2014.2376876

[14] Tang X, Falco G, Falletti E, Presti LL. Complexity reduction of the Kalman filter-based tracking loops in GNSS receivers. GPS Solutions. 2017;21:685-699. DOI: 10.1007/s10291016-0557-6

[15] Jatoth RK, Gopisetty S, Hussai M. Performance analysis of alpha beta filter, Kalman filter and meanshift for object tracking in video sequences. International Journal of Image, Graphics and Signal Processing. 2015;3:24-30. DOI: 10.5815/ijigsp.2015.03.04

[16] Li Z, Wu H. A survey of Maneuvering target tracking using Kalman filter. Proc. 4th Int. Conf. Mechatronics Mater. Chem. Comput. Eng. (ICMMCCE). 2015;39:542-545. DOI: 10.2991/icmmcce-15.2015.109

[17] Saho K, Homma H, Sakamoto T, Sato T, Inoue K, Fukuda T. Accurate image separation method for two closely spaced pedestrians using UWB Doppler imaging radar and supervised learning. IEICE Transactions on Communications. 2014;97:1223-1233. DOI: 10.1587/transcom.E97.B.1223

[18] Anabuki M, Okumura S, Sato T, Sakamoto T, Saho K, Yoshioka M, Inoue K, Fukuda T, Sakai H. Ultrawideband radar imaging using adaptive Array and Doppler separation. IEEE Transactions on Aerospace and Electronic Systems. 2017;53:190-200. DOI: 10.1109/ TAES.2017.2649798

[19] Cossaboom M, Georgy J, Karamat T, Noureldin A. Augmented kalman filter and map matching for 3D RISS/GPS integration for land vehicles. International Journal of Navigation and Observation. 2012;2012:576807. DOI: 10.1155/2012/576807 
[20] Afia AB, Escher AC, Macabiau C, Roche S. A GNSS/IMU/WSS/VSLAM hybridization using an extended Kalman filter. In: Proc. of ION 2015 Pacific PNT Meeting. 2015. pp. 719-732

[21] Kellner D, Barjenbruch M, Klappstein J, Dickmann J, Dietmayer K. Tracking of extended objects with high-resolution Doppler radar. IEEE Transaction on Intelligent Transportation Systems. 2016;17:1341-1353. DOI: 10.1109/TITS.2015.2501759

[22] Carrolla P, Domresea K, Zhoub H, Zhoua S, Willetta P. Doppler-aided localization of mobile nodes in an underwater distributed antenna system. Physical Communication. 2016;18:49-59. DOI: 10.1016/j.phycom.2015.08.008

[23] Zhoua G, Wub L, Xiea J, Denga W, Quan T. Constant turn model for statically fused converted measurement Kalman filters. Signal Processing. 2015;108:400-411. DOI: 10.1016/ j.sigpro.2014.09.022

[24] Cho MH, Tahk MJ, Kim MC, Lee D, Yoon J. Modified gain pseudo-measurement filter design for radar target tracking with range rate measurement. In: Proc. IEEE 2017 25th Mediterranean Conf. on Control and Automation (MED). 2017. pp. 1195-1200. DOI: 10.1109/ MED.2017.7984280

[25] Saho K, Masugi M. Performance analysis of fixed-gain position-velocity-measured tracking filter. In: 2016 2nd International Conference on Control, Automation and Robotics (ICCAR); 28-30 April 2016; Hong Kong, China. IEEE; 2016. DOI: 10.1109/ICCAR.2016. 7486760

[26] Saho K, Masugi M. Performance analysis of alpha-beta-gamma tracking filters using position and velocity measurements. EURASIP Journal on Advances in Signal Processing. 2015;2015:35. DOI: 10.1186/s13634-015-0220-3

[27] Kalata PR. The tracking index: A generalized parameter for alpha-beta and alpha-betagamma target trackers. IEEE Transactions on Aerospace and Electronic Systems. 1984; AES-20:174-182. DOI: 10.1109/TAES.1984.310438 
